M. Kurata

Nagoya Math. J.

Vol. 74 (1979), 77-86

\title{
HYPERBOLIC NONWANDERING SETS WITHOUT DENSE PERIODIC POINTS
}

\author{
MASAHIRO KURATA
}

In this paper we give a negative answer to the problem which is suggested in [3]: if a nonwandering set $\Omega$ is hyperbolic, are the periodic points dense in $\Omega$ ?

Newhouse and Palis proved that on two dimensional closed manifolds the answer is positive ([1], [2]).

Suppose that $f: M \rightarrow M$ is a diffeomorphism of a manifold $M$. A point $x \in M$ is a nonwandering point of $f$ if for any neighbourhood $U \subset M$ of $x$ there is a positive integer $n$ such that $f^{n}(U) \cap U \neq \varnothing . \quad \Omega=$ nonwandering points of $f\}$ is called the nonwandering set of $f$. A point of $M-\Omega$ is a wandering point. A nonwandering set $\Omega$ of $f$ is hyperbolic if $\Omega$ is compact and $T M \mid \Omega$ splits into a Whitney sum of $T f$-invariant subbundles

$$
T M \mid \Omega=E^{s} \oplus E^{u},
$$

and there are $c>0,0<\lambda<1$ such that

$$
\left\|T f^{n} v\right\| \leqq c \lambda^{n}\|v\| \quad \text { if } v \in E^{s}
$$

and

$$
\left\|T f^{-n} v\right\| \leqq c \lambda^{n}\|v\| \quad \text { if } v \in E^{u}
$$

for $n>0$.

We will prove the following.

THEOREm. Suppose that $M$ is a manifold with $\operatorname{dim} M \geqq 4$. Then there is a diffeomorphism $F: M \rightarrow M$ such that the nonwandering set $\Omega$ is hyperbolic but periodic points of $F$ are not dense in $\Omega$.

Proof. 0. An outline of Proof. To simplify the proof, we assume $\operatorname{dim} M=4$. In 1 we construct an embedding of 2 -dimensional disk $f: D$

Received February 20, 1978. 
$\rightarrow D$, where $f$ has a hyperbolic set consisting of finite fixed points and two non-periodic orbits $\mathcal{O}_{1}$ and $\mathcal{O}_{2}(13.2,13.3)$. In $2 \sim 10$ we will extend $f$ to an embedding $F: N \rightarrow N$, where $M \supset N=D \times D^{2} \cup 1$-handle. The nonwandering set of $F$ consists of a finite number of fixed points and the two orbits $\mathcal{O}_{1}, \mathcal{O}_{2}$, where points nearby $\mathcal{O}_{1}$ (resp. $\mathcal{O}_{2}$ ) return near by $\mathcal{O}_{1}$ (resp. $\mathcal{O}_{2}$ ) through the 1-handle (15). And other points are wandering (12, 14). Finally we extend $F$ to a diffeomorphism of $M$.

1.

Let

$$
D=[-2,6] \times[-1,3] \subset R^{2}
$$

and an embedding $f: D \rightarrow D$ satisfy the followings (Figure 1). Suppose that real numbers $a_{-1}, \cdots, a_{6}$ satisfy

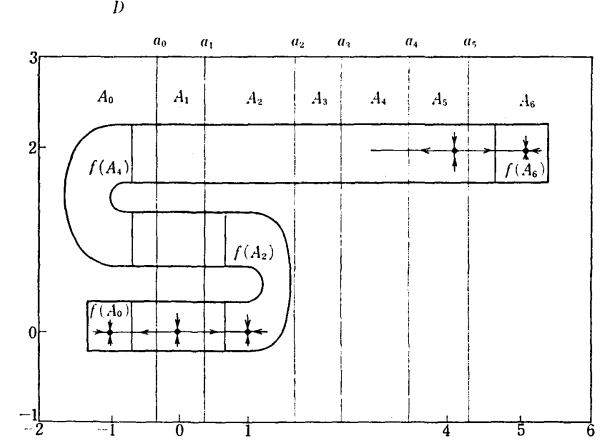

Figure 1

$$
\begin{aligned}
a_{-1}= & -2<-1<a_{0}=-a_{1}<0<a_{1}<1<a_{2}<a_{3} \\
& <a_{4}<4<a_{5}<5<a_{6}=6,
\end{aligned}
$$

and the rectangle $A_{i}(i=0, \cdots, 6)$ is given by

$$
A_{i}=\left\{(x, y) \in D \mid a_{i-1} \leqq y \leqq a_{i}\right\} .
$$

Then $f$ satisfies $(1.2) \sim(1.5)$.

(1.2) $f\left|A_{0}, f\right| A_{2}$ and $f \mid A_{6}$ are contractions with three sinks $(-1,0)$, $(1,0),(5,2)$,

(1.3) $f\left(A_{4}\right) \subset \operatorname{int} A_{0}$

(1.4) $f \mid A_{i}: A_{i} \rightarrow f\left(A_{i}\right)(i=1,3,5)$ maps $A_{i}$ linearly onto a rectangle $f\left(A_{i}\right)$, expanding the $x$-direction and contracting the $y$-direction. There are two hyperbolic fixed points $(0,0)$ and $(4,2)$. 
(1.5) There are numbers $\alpha>1$ and $0<\beta<1$ such that

$$
f(x, y)= \begin{cases}(\alpha x, \beta y) & \text { for }(x, y) \in A_{1} \\ (\alpha(x-4)+4, \beta(y-2)+2) & \text { for }(x, y) \in A_{5}\end{cases}
$$

2.

Let $D^{\prime} \subset R^{2}$ satisfy the followings (Figure 2). $\quad D^{\prime}$ is a neighbourhood of $(\{0\} \times[-1,1]) \cup([-2,0] \times\{0\})$ which is diffeomorphic to a 2-dimensional disk, and there is a sufficiently small positive number $\varepsilon$ such that

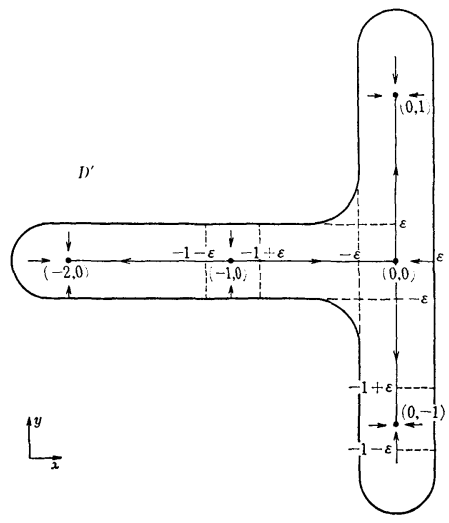

Figure 2

$$
\left\{(x, y) \in D^{\prime}|| y+1 \mid \leqq \varepsilon\right\}=[-\varepsilon, \varepsilon] \times[-1-\varepsilon,-1+\varepsilon] .
$$

and

$$
\left\{(x, y) \in D^{\prime}|| x+1 \mid \leqq \varepsilon\right\}=[-1-\varepsilon,-1+\varepsilon] \times[-\varepsilon, \varepsilon] .
$$

Let an embedding $g: D^{\prime} \rightarrow D^{\prime}$ satisfy $(2.1) \sim(2.9)$.

(2.1) $g\left(D^{\prime}\right) \subset \operatorname{int} D^{\prime}$

(2.2) $g$ is isotopic to the identity,

(2.3) $\bigcup_{n>0} g^{n}\left(D^{\prime}\right)=(\{0\} \times[-1,1]) \cup([-2,0] \times\{0\})$,

(2.4) there are five fixed points, that is, three sinks $(-2,0),(0,1)$, $(0,-1)$, and two saddle points $(0,0),(-1,0)$,

(2.5) $W^{u}((0,0))=\{0\} \times(-1,1)$,

(2.6) $W^{u}((-1,0))=(-2,0) \times\{0\}$,

(2.7) $W^{s}((0,0)) \cap D^{\prime}=\left\{(x, 0) \in D^{\prime} \mid-1<x\right\}$,

where $W^{s}(p)$ (resp. $\left.W^{u}(p)\right)$ is the stable (resp. unstable) manifold through p. $(-1,1)$ and $(-2,0)$ denote open intervals.

(2.8) $g(x, y)=\left(\frac{1}{2} x, \frac{1}{2}(y+1)-1\right)$ if $|y+1| \leqq \varepsilon$, 
(2.9) $g(x, y)=\left(2(x+1)-1, \frac{1}{2} y\right)$ if $|x+1| \leqq \varepsilon$.

3.

\section{Define}

$$
N=D \times D^{\prime} \cup_{\psi} D^{3}(\delta) \times[0,1],
$$

where

$$
D^{3}(\delta)=\left\{\left(y_{1}, y_{2}, y_{3}\right) \in \boldsymbol{R}^{3} \mid \sqrt{y_{1}^{2}+y_{2}^{2}+y_{3}^{2}} \leqq \delta\right\}
$$

and

$$
0<\delta<\frac{1}{4} \varepsilon
$$

The attaching map

$$
\psi: D^{3}(\delta) \times([0, \varepsilon] \cup[1-\varepsilon, 1]) \rightarrow D \times D^{\prime}
$$

is given by

$$
\psi\left(y_{1}, y_{2}, y_{3}, t\right)= \begin{cases}\left(y_{1}, y_{2}, t, y_{3}-1\right) & \text { if } 0 \leqq t \leqq \varepsilon \\ \left(y_{1}+5, y_{2}+2, y_{3}-1,1-t\right) & \text { if } 1-\varepsilon \leqq t \leqq 1\end{cases}
$$

(Figure 3).

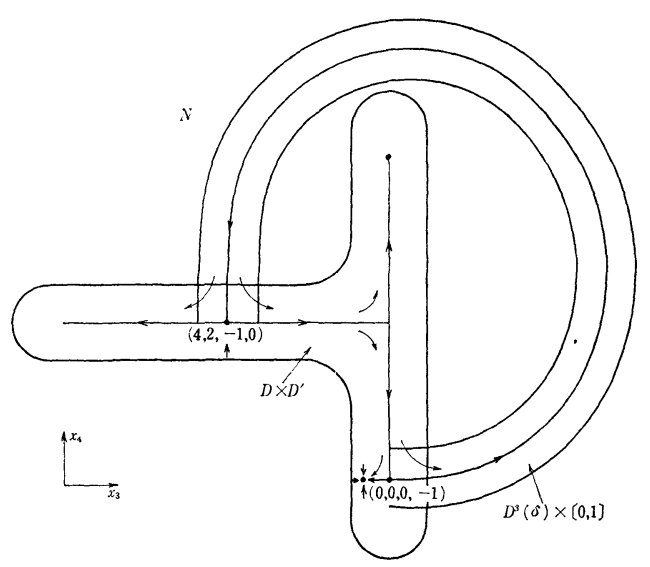

Figure 3

In $4 \sim 10$, we will construct an embedding $F: N \rightarrow N$. After this, $\left(x_{1}, x_{2}, x_{3}, x_{4}\right)$ (resp. $\left.\left(y_{1}, y_{2}, y_{3}, t\right)\right)$ denotes a point of $D \times D^{\prime} \subset N$ (resp. $D^{3}(\delta)$ $\times[0,1] \subset N)$. 
4.

For $\left(x_{1}, x_{2}, x_{3}, x_{4}\right) \in D \times D^{\prime}$ with $\left|x_{3}+1\right| \geqq \varepsilon$ and $\left|x_{4}+1\right| \geqq \varepsilon$, define (4.1) $\quad F\left(x_{1}, x_{2}, x_{3}, x_{4}\right)=\left(f\left(x_{1}, x_{2}\right), g\left(x_{3}, x_{4}\right)\right)$.

5.

For $\left(x_{1}, x_{2}, x_{3}, x_{4}\right) \in D \times D^{\prime}$ with $\frac{1}{4} \varepsilon \leqq\left|x_{4}+1\right| \leqq \varepsilon$, define

(5.1) $F\left(x_{1}, x_{2}, x_{3}, x_{4}\right)=\left(f_{\left|x_{4}+1\right|}\left(x_{1}, x_{2}\right), g\left(x_{3}, x_{4}\right)\right)$, where $f_{t}: D \rightarrow D(0 \leq t \leq \varepsilon)$ is an isotopy satisfying (5.2) (5.6). Suppose that $b_{i}(i=1, \cdots, 4)$ is a positive number with

$$
0<b_{1}<b_{2}<\delta<b_{3}<b_{4}<a_{1}, \quad \alpha b_{1}<b_{2},
$$

and

$$
b_{4}<\min \left\{4-a_{4}, a_{5}-4\right\}
$$

Then

$$
\begin{gathered}
f_{t}\left(x_{1}, x_{2}\right)=f\left(x_{1}, x_{2}\right) \quad \text { if }\left|x_{1}\right|<b_{1} \text { or }\left|x_{1}\right|>b_{4}, \\
f_{t}=f \quad \text { for } \frac{1}{2} \varepsilon \leqq t \leqq \varepsilon, \\
f_{t}=f_{0} \quad \text { for } 0 \leqq t \leqq \frac{1}{4} \varepsilon,
\end{gathered}
$$

and

$$
f_{t}\left(x_{1}, x_{2}\right)=\left(\bar{f}_{t}\left(x_{1}\right), \beta x_{2}\right) \quad \text { for }\left|x_{1}\right| \leqq b_{4},
$$

where $\bar{f}_{t}$ is an isotopy of a neighbourhood of 0 in $\boldsymbol{R}^{1}$ and $\bar{f}_{0}$ has five fixed points: three sources $0, \pm b_{3}$, and two sinks $\pm b_{2}$ (Figure 4 ).

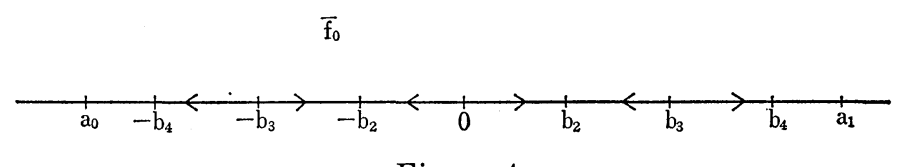

Figure 4

6.

For $\left(x_{1}, x_{2}, x_{3}, x_{4}\right) \in D \times D^{\prime}$ with $\left|x_{4}+1\right|<\frac{1}{4} \varepsilon, F$ is defined as follows. Let

$$
U=\left\{\left(x_{1}, x_{2}, x_{3}, x_{4}\right) \in D \times D^{\prime} \mid \sqrt{x_{1}^{2}+x_{2}^{2}+\left(x_{4}+1\right)^{2}} \leqq \delta\right\},
$$

and

$$
U_{1}=\left\{\left(x_{1}, x_{2}, x_{3}, x_{4}\right) \in D \times D^{\prime} \mid \sqrt{x_{1}^{2}+x_{2}^{2}+\left(x_{4}+1\right)^{2}} \leqq \delta_{1}\right\},
$$


where $b_{2}<\delta_{1}<\delta$. Then $F$ is defined as follows.

$$
\begin{aligned}
F\left(x_{1}, x_{2}, x_{3}, x_{8}\right)= & \left(f_{0}\left(x_{1}, x_{2}\right), g\left(x_{3}, x_{4}\right)\right) \\
& \text { if }\left(x_{1}, x_{2}, x_{3}, x_{4}\right) \in D \times D^{\prime}-U \text { and }\left|x_{4}+1\right| \leqq \frac{1}{4} \varepsilon,
\end{aligned}
$$

$F$ is written in the form such that

$$
\begin{aligned}
F\left(x_{1}, x_{2}, x_{3}, x_{4}\right)= & \left(f_{0}\left(x_{1}, x_{2}\right), \bar{g}\left(x_{1}, x_{2}, x_{3}, x_{4}\right), \frac{1}{2}\left(x_{4}+1\right)-1\right) \\
& \text { if }\left(x_{1}, x_{2}, x_{3}, x_{4}\right) \in U \text { and } x_{3}>-\frac{1}{2} \varepsilon,
\end{aligned}
$$

where $\bar{g}$ satisfies the followings.

$$
\begin{gathered}
\bar{g}\left(x_{1}, x_{2}, x_{3}, x_{4}\right)=\frac{1}{2} x_{3} \quad \text { near the frontier of } U, \\
\bar{g}\left(x_{1}, x_{2}, x_{3}, x_{4}\right)=2 x_{3} \text { if }\left(x_{1}, x_{2}, x_{3}, x_{4}\right) \in U_{1} \text { and }-\frac{1}{4} \varepsilon \leqq x_{3}<\frac{1}{2} \varepsilon,
\end{gathered}
$$

and

(6.7) $\bar{g}\left(x_{1}, x_{2}, x_{3}, x_{4}\right)$ does not depend on $x_{1}$ if $\left|x_{1}\right|<b_{1}$. $\bar{g}$ as above can be induced from a vector field ( $b_{1}$ is assumed to be sufficiently small).

$$
\begin{aligned}
& F\left(\left\{\left(x_{1}, x_{2}, x_{3}, x_{4}\right) \in U \mid x_{3}<0\right\}\right) \\
& \subset\left\{\left(x_{1}, x_{2}, x_{3}, x_{4}\right) \in U \mid x_{3}<0\right\} .
\end{aligned}
$$

In $\left\{\left(x_{1}, x_{2}, x_{3}, x_{4}\right) \in U \mid x_{3}<0\right\}$ there are only a finite number of nonwandering points, which are hyperbolic fixed points. Furthermore $F$ satisfies the conditions in 10 .

7.

On $D^{3}(\delta) \times[0,1-\varepsilon], F$ is given as follows.

$$
F\left(y_{1}, y_{2}, y_{3}, t\right)=\left(f_{0}\left(y_{1}, y_{2}\right), \frac{1}{2} y_{3}, \phi\left(y_{1}, y_{2}, y_{3}, t\right)\right),
$$

where $\phi$ satisfies the followings.

If $\sqrt{y_{1}^{2}+y_{2}^{2}+y_{3}^{2}}<\delta_{1}$ or $\frac{1}{2}<t$

(7.2) $\phi\left(y_{1}, y_{2}, y_{3}, t\right)$ depends only on $t$,

and

$$
\begin{gathered}
\frac{d \phi}{d t}>0 \\
\phi\left(y_{1}, y_{2}, y_{3}, t\right)=1-\frac{1}{2}(1-t) \quad \text { for } 1-2 \varepsilon \leqq t \leqq 1-\varepsilon . \\
\phi\left(y_{1}, y_{2}, y_{3}, t\right)=\bar{g}\left(y_{1}, y_{2}, t, y_{3}-1\right) \quad \text { if } 0 \leqq t \leqq \varepsilon .
\end{gathered}
$$

Moreover $F$ satisfies 10. 
8.

For $\left(x_{1}, x_{2}, x_{3}, x_{4}\right) \in D \times D^{\prime}$ with $\left|x_{3}+1\right|<\frac{1}{4} \varepsilon, F$ is given as follows. Let $h_{t}: D \rightarrow D(0 \leqq t \leqq \varepsilon)$ be an isotopy such that

$$
h_{t}=f \quad \text { if } \frac{1}{2} \varepsilon \leqq t \leqq \varepsilon,
$$

$$
h_{t}\left(x_{1}, x_{2}\right)=f\left(x_{1}, x_{2}\right) \quad \text { if }-2 \leqq x_{1} \leqq 4-b_{4} \text { or } 4+b_{4} \leqq x_{1} \leqq 6,
$$

and

$$
h_{t}\left(x_{1}, x_{2}\right)=f_{t}\left(x_{1}-4, x_{2}-2\right)+(4,2) \quad \text { if }\left|x_{1}-4\right|<b_{4} .
$$

Then $F$ is written in the form such that

$$
F\left(x_{1}, x_{2}, x_{3}, x_{4}\right)=\left(h_{0}\left(x_{1}, x_{2}\right), \bar{h}\left(x_{1}, x_{2}, x_{3}, x_{4}\right), \frac{1}{2} x_{4}\right),
$$

where $\bar{h}$ satisfies the followings.

$$
\begin{aligned}
\bar{h}\left(x_{1}, x_{2}, x_{3}, x_{4}\right)= & \frac{1}{2}\left(x_{3}+1\right)-1 \\
& \text { if } \sqrt{\left(x_{1}-4\right)^{2}+\left(x_{2}-2\right)^{2}+\left(x_{3}+1\right)^{2}} \leqq \delta \text { and } x_{4}>\frac{2}{3} \varepsilon, \\
\bar{h}\left(x_{1}, x_{2}, x_{3}, x_{4}\right)= & 2\left(x_{3}+1\right)-1 \\
& \text { if } \sqrt{\left(x_{1}-4\right)^{2}+\left(x_{2}-2\right)^{2}+\left(x_{3}+1\right)^{2}} \geqq \delta_{2} \text { or } x_{4}<\frac{1}{3} \varepsilon,
\end{aligned}
$$

where $\delta<\delta_{2}<\frac{1}{4} \varepsilon$.

(8.7) $\bar{h}\left(x_{1}, x_{2}, x_{3}, x_{4}\right)$ does not depend on $x_{1}$ if $\left|x_{1}-4\right|<b_{1}$. Furthermore $F$ satisfies 10 .

9.

For $\left(x_{1}, x_{2}, x_{3}, x_{4}\right) \in D \times D^{\prime}$ with $\frac{1}{4} \varepsilon \leqq\left|x_{3}+1\right|<\varepsilon$, define

$$
F\left(x_{1}, x_{2}, x_{3}, x_{4}\right)=\left(h_{\left|x_{3}+1\right|}\left(x_{1}, x_{2}\right), 2\left(x_{3}+1\right)-1, \frac{1}{2} x_{4}\right) \text {. }
$$

10.

$F$ is an embedding of $N$ such that

and

$$
F(N) \subset \operatorname{int} N,
$$

(10.2) $F$ is isotopic to the identity.

11.

Straightening the corner (and modifying $F$ near the corner), we can regard $N$ as a submanifold of $M$ which is diffeomorphic to $D^{3} \times S^{1}$. Extend $F$ to a diffeomorphism of $M$ such that the nonwandering set of 
$F \mid M-N$ consists of a finite number of hyperbolic fixed points.

12.

In $12 \sim 15$, we will show that the number of nonwandering points of $F \mid N$ is countably infinite. This implies that the diffeomorphism of $M$ as above is the required one, because its periodic points are finite. (12.1) and (12.2) follow from the construction of $F$.

(12.1) If $\left(x_{3}, x_{4}\right) \notin(\{0\} \times[1,-1]) \cup([-2,0] \times\{0\})$, then $\left(x_{1}, x_{2}, x_{3}, x_{4}\right) \in N$ is a fixed point or a wandering point.

(12.2) If $\left(x_{1}, x_{2}\right) \neq(0,0)$, then $\left(x_{1}, x_{2}, x_{3}, x_{4}\right) \in N$ is a fixed point or a wandering point.

13.

The maximal invariant set of $F \mid(D \times\{0\} \times\{0\})$ consists of points satisfying one of the conditions $(13.1) \sim(13.3)$.

(13.1) $\left(x_{1}, x_{2}, 0,0\right) \in D \times D$ such that there is an integer $n_{0}$ with

$$
f^{n}\left(x_{1}, x_{2}\right) \in A_{i}(0 \leqq i \leqq 6) \quad \text { for } n \in Z
$$

and

$$
f^{n}\left(x_{1}, x_{2}\right) \in A_{i}(i=0,2,5,6) \quad \text { for } n>n_{0},
$$

where $Z$ denotes the integers.

(13.2) $\left(x_{1}, x_{2}, 0,0\right) \in D \times D^{\prime}$ such that there is $n_{0} \in Z$ with

$$
\begin{array}{ll}
f^{n}\left(x_{1}, x_{2}\right) \in A_{5} & \text { if } n<n_{0} \\
f^{n}\left(x_{1}, x_{2}\right) \in A_{3} & \text { if } n=n_{0}
\end{array}
$$

and

$$
f^{n}\left(x_{1}, x_{2}\right) \in A_{1} \quad \text { if } n>n_{0} .
$$

(13.3) $\left(x_{1}, x_{2}, 0,0\right) \in D \times D^{\prime}$ such that there is $n_{0} \in Z$ with

$$
f^{n}\left(x_{1}, x_{2}\right) \in A_{5} \quad \text { for } n<n_{0}
$$

and

$$
f^{n}\left(x_{1}, x_{2}\right) \in A_{1} \quad \text { for } n \geqq n_{0} \text {. }
$$

Denote

$$
\mathcal{O}_{1}=\left\{\left(x_{1}, x_{2}, 0,0\right) \in D \times D^{\prime} \mid\left(x_{1}, x_{2}, 0,0\right) \text { satisfies }(13.2)\right\}
$$


and

$$
\mathcal{O}_{2}=\left\{\left(x_{1}, x_{2}, 0,0\right) \in D \times D^{\prime} \mid\left(x_{1}, x_{2}, 0,0\right) \text { satisfies (13.3) }\right\}
$$

Then $\mathcal{O}_{i}(i=1,2)$ is an orbit of one point, and $\mathcal{O}_{1} \cup \mathcal{O}_{2} \cup\{(4,2,0,0),(0,0$, $0,0)\}$ is a hyperbolic set.

14 .

We will show that any point satisfying (13.1) is a fixed point or a wandering point. Suppose $\left(x_{1}, x_{2}, 0,0\right)$ is not a fixed point and satisfies (13.1). Let $W_{1} \subset D$ be a neighbourhood of $\left(x_{1}, x_{2}\right)$ with

$$
f^{n}\left(W_{1}\right) \subset A_{i}(i=0,26) \quad \text { for } n>n_{0},
$$

where $n_{0}$ is given in (13.1). (It does not occur that $f^{n}\left(x_{1}, x_{2}\right) \in A_{5}$ for $n>n_{0}$, because $\left(x_{1}, x_{2}\right) \neq(4,2)$.) Choose a neighbourhood $W_{2} \subset D^{\prime}$ of $(0,0)$ such that

$$
g^{n}\left(W_{2}\right) \cap\left\{\left(x_{3}, x_{4}\right) \in D^{\prime}|| x_{4}+1 \mid \leqq \varepsilon\right\}=\varnothing \quad \text { for } n \leqq n_{0} .
$$

If $n \leqq n_{0}$ (resp. $n>n_{0}$ ), it follows from (14.2) (resp. (14.1) and (5.3)) that

$$
\begin{array}{r}
F^{n}(w) \cap\left\{\left(z_{1}, z_{2}, z_{3}, z_{4}\right) \in D \times D^{\prime} \mid \sqrt{z_{1}^{2}+z_{2}^{2}+\left(z_{3}+1\right)^{2}}<\delta\right\}=\varnothing \\
\text { for } w \in W_{1} \times W_{2} .
\end{array}
$$

Therefore $\left(x_{1}, x_{2}, 0,0\right)$ is a wandering point, because a non-periodic point is nonwandering only if its nearby points return near by the point through $D^{3}(\delta) \times[0,1]$.

15.

Here we will prove that a point of $\mathcal{O}_{1} \cup \mathcal{O}_{2}$ is nonwandering. Suppose that $\left(x_{1}, x_{2}, 0,0\right) \in D \times D^{\prime}$ satisfies (13.2) or (13.3). Let $W$ be a neighbourhood of $\left(x_{1}, x_{2}, 0,0\right)$ such that

$$
\begin{array}{r}
W=\left\{\left(z_{1}, z_{2}, z_{3}, z_{4}\right) \in D \times D^{\prime}|| z_{i}-x_{i}|\leqq \sigma,| z_{j} \mid \leqq \sigma\right. \\
\text { for } i=1,2, j=3,4\}
\end{array}
$$

for $0<\sigma<b_{1}$. Choose a sequence $\left(x_{1}, x_{2}, y_{3}^{(i)}, y_{4}^{(i)}\right) \in W(i=1,2, \cdots)$ with

$$
y_{3}^{(i)}>0, \quad y_{4}^{(i)}<0,
$$

and

$$
y_{3}^{(i)} \rightarrow 0, \quad y_{4}^{(i)} \rightarrow 0 \quad(\text { as } i \rightarrow \infty)
$$


Then there is a sequence of integers $\left\{n_{i}\right\}_{i=1,2}, \ldots$ which satisfies $n_{i} \rightarrow \infty$ (as $i \rightarrow \infty)$ and

$$
F^{n_{i}}\left(x_{1}, x_{2}, y_{3}^{(i)}, y_{4}^{(i)}\right) \rightarrow(0,0,0,-1) \quad \text { as } i \rightarrow \infty \text {. }
$$

This implies that there is a sequence $\left\{m_{i}\right\}_{i=1,2, \ldots}$ with $m_{i}>n_{i}$ and

$$
F^{m_{i}}\left(x_{1}, x_{2}, y_{3}^{(i)}, y_{4}^{(i)}\right) \rightarrow(4,2,-1,0) \quad \text { as } i \rightarrow \infty \text {. }
$$

This implies that there is a sequence $\left\{\ell_{i}\right\}_{i=1,2, \ldots}$ with $\ell_{i}>m_{i}$ and

$$
F^{\ell_{i}}\left(x_{1}, x_{2}, y_{3}^{(i)}, y_{4}^{(i)}\right) \rightarrow(4,2,0,0) \quad \text { as } i \rightarrow \infty \text {. }
$$

It follows from (1.5), (4.1), (5.1), (5.3), (6.3), (6.4), (6.7), (7.1), (7.2), (8.3), (8.4),

(8.7) and (9.1) that on a neighbourhood of $(4,2,0,0) F$ satisfies

$$
\begin{aligned}
F^{\ell_{i}}\left(\left[x_{1}-\sigma, x_{1}+\sigma\right] \times\left\{x_{2}\right\} \times\left\{y_{3}^{(i)}\right\} \times\left\{y_{4}^{(i)}\right\}\right) \\
\supset[4-\sigma, 4+\sigma] \times\left\{v_{2}^{(i)}\right\} \times\left\{v_{3}^{(i)}\right\} \times\left\{v_{4}^{(i)}\right\},
\end{aligned}
$$

where

$$
v_{j}^{(i)}=\operatorname{pr}_{j} F^{\ell_{i}}\left(x_{1}, x_{2}, y_{3}^{(i)}, y_{4}^{(i)}\right),
$$

and $p r_{j}$ is the projection to the $x_{j}$-factor. It follows from (15.5) and (15.6) that for sufficiently large $k$

$$
\bigcup_{i>0} F^{\ell_{i}+k}\left(\left[x_{1}-\sigma, x_{1}+\sigma\right] \times\left\{x_{2}\right\} \times\left\{y_{3}^{(i)}\right\} \times\left\{y_{4}^{(i)}\right\}\right) \cap W \neq \varnothing .
$$

Thus $\left(x_{2}, x_{2}, 0,0\right)$ is a nonwandering point. We have constructed $F$ such that $\mathcal{O}_{1} \cup \mathcal{O}_{2} \cup\{$ fixed points $\}$ is hyperbolic. This completes the proof.

After this paper was written the author was informed that A. Danker also constructed a counter-example to this problem. (c.f. On Smale's Axiom $A$ dynamical systems, Ann. of Math. 107 (1978) 517-553.)

\section{References}

[1] S. Newhouse and J. Palis, Hyperbolic nonwandering sets on two-dimensional manifolds, Dynamical Systems, ed. M. M. Peixoto, Academic Press, 1973.

[ 2 ] J. Palis and C. C. Pugh, Fifty problems in dynamical systems, Dynamical Systems -Warwick 1974, ed. A. Manning, Lecture Notes in Math., 468, Springer-Verlag, 1975.

[ 3 ] S. Smale, Differentiable dynamical systems, Bull. Amer. Math. Soc., 73 (1967), 747-817.

Department of Mathematics

Faculty of Science

Hokkaido University

Current Address:

Department of Mathematics

Nagoya Institute of Technology 